\title{
Caracterización biogeográfica de la Faja Volcánica Transmexicana y análisis de los patrones de distribución de su mastofauna
}

\section{Biogeographic characterization of the Transmexican Volcanic Belt and analysis of the distributional patterns of the mammal fauna}

\author{
Niza Gámez ${ }^{1 凶}$, Tania Escalante ${ }^{1}$, Gerardo Rodríguez ${ }^{2}$, Miguel Linaje ${ }^{3}$ y Juan J. Morrone ${ }^{1}$ \\ ${ }^{1}$ Museo de Zoología Alfonso L. Herrera, Departamento de Biología Evolutiva, Facultad de Ciencias, Universidad Nacional Autónoma de México. \\ Apartado postal 70-399, 04510 México, D. F., México. \\ ${ }^{2}$ Unidad de Geomática, Instituto de Ecología, Universidad Nacional Autónoma de México. Apartado postal 70-275, 04510 México, D. F., México. \\ ${ }^{3}$ Laboratorio de Sistemas de Información Geográfica, Instituto de Biología, Universidad Nacional Autónoma de México. Apartado postal 70-153, \\ 04510 México, D. F., México. \\ 凶izagt@gmail.com
}

\begin{abstract}
Resumen. La provincia de la Faja Volcánica Transmexicana (FVT) está reconocida como centro de diversificación, endemismo y transición biogeográfica. Debido a su heterogeneidad ambiental, origen geológico complejo e intrincados patrones de distribución, aún no existe acuerdo en cuanto a su delimitación geográfica y la diferenciación ecológica y biogeográfica de su biota. Para realizar la caracterización de la provincia y de las unidades que la conforman y analizar los patrones de distribución de su mastofauna, a partir de los límites geográficos y lista de especies, se realizó un análisis espacial de su geología, altitud, clima y tipo de vegetación. Asimismo, se analizaron los patrones de riqueza y endemismo asociados con diferentes variables ambientales; esto último, a partir de modelos de nicho ecológico. Se caracteriza la FVT como una unidad biogeográfica con 2 distritos (este y oeste), donde la vegetación y la altitud son las variables que mejor explican la distribución de riqueza y endemismo de su mastofauna y la porción más relevante el bosque de pino-encino de los 2000 a los 3000 metros. La mayor riqueza de especies se presenta en los órdenes Rodentia y Chiroptera; 12 de los 13 géneros de mamíferos endémicos de México tienen representación en la FVT y se registran 14 especies endémicas de la provincia, en su mayoría roedores.
\end{abstract}

Palabras clave: biogeografía, Mammalia, provincia biótica, riqueza, modelos de nicho ecológico, Eje Neovolcánico.

\begin{abstract}
The Transmexican Volcanic Belt (TVB) is recognized as a center of diversification, endemism and biogeographic transition. Due to its environmental heterogeneity, complex geological origin and intricate distributional patterns, there is no consensus on its geographic delimitation and the ecological and biogeographic differentiation of its biota. We undertook a spatial analysis of the geology, altitude, climate and vegetation types, and the richness and endemicity patterns associated, with the aim of characterizing the province and the units within it. We also analyzed the distributional patterns of the mammal fauna, based on ecological niche models of the species. The TVB is characterized as a biogeographic unit, with 2 districts (east and west), where vegetation and altitude explain the distribution of richness and endemism of the mammal fauna, and the pine-oak forest from 2000 to $3000 \mathrm{~m}$ is the most relevant portion. The highest species richness is found in orders Rodentia and Chiroptera; 12 out of the 13 mammal genera endemic to Mexico are represented in the TVB, and 14 endemic species are registered for the province, mostly rodents.
\end{abstract}

Key words: biogeography, Mammalia, biotic province, richness, ecological niche models, Neovolcanic Belt.

\section{Introducción}

En la naturaleza, las especies no están distribuidas al azar, sino que exhiben patrones de distribución donde taxones diferentes se encuentran en un mismo espacio y tiempo como resultado de procesos históricos y ecológicos comunes (Morrone, 2009). El reconocimiento de las unida-

Recibido: 10 junio 2010; aceptado: 02 agosto 2011 des biogeográficas conocidas como componentes bióticos brinda información sobre el proceso evolutivo de las biotas y de las áreas que ocupan. Para que estas unidades constituyan entidades naturales, deben estar sustentadas por patrones de homología biogeográfica, es decir, basarse en la distribución simpátrida de especies endémicas, que pese a tener historias de vida y relaciones filogenéticas independientes, están en simpatría como resultado de procesos biogeográficos comunes (Morrone, 2001, 2004; Escalante, 2009). De manera similar a la sistemática, donde las homo- 
logías sucesivamente anidadas permiten el reconocimiento de esquemas jerarquizados, el reconocimiento de homologías biogeográficas sucesivamente anidadas permite proponer esquemas de regionalización biogeográfica (Escalante, 2009).

La gran complejidad biogeográfica del territorio mexicano ha promovido el establecimiento de múltiples esquemas de regionalización en los que se reconoce la existencia de una unidad biogeográfica correspondiente a la provincia de la Faja Volcánica Transmexicana (FVT) (Simpson, 1964; Wilson, 1974; Pagel et al., 1991; Kaufman, 1995; Brown y Lomolino, 1998; Morrone, 2005, 2010). La FVT es un conjunto de cordilleras de volcanes alineados sobre una franja que cruza el territorio mexicano de oeste a este, desde Cabo Corrientes, Nayarit hasta la sierra Chinconquiaco, Veracruz (Ferrusquía-Villafranca, 2007), que se localiza principalmente entre los 19 y $21^{\circ} \mathrm{de}$ latitud norte y entre los 1500 y 3000 m de altitud (Ferrusquía-Villafranca, 2007). Esta unidad se reconoce como centro de diversificación, endemismo y transición biogeográfica para una gran variedad de taxones (Smith, 1941; Goldman y Moore, 1946; Halffter, 1976; Ramamoorthy et al., 1998; Challenger, 1998; Morrone, 2005, 2010). La faja de volcanes que conforman la FVT, aún en actividad, se ha formado durante los últimos 19 millones de años, a lo largo de 4 grandes episodios de vulcanismo que afectaron de manera asincrónica el territorio, primero en el oeste y tardíamente en el este; sin embargo, los más prominentes se produjeron en los últimos 3 millones de años (Espinosa y Ocegueda, 2007). Esta asincronía geológica se ve igualmente reflejada en la distribución no homogénea de su riqueza y endemismos (Villaseñor y Ortiz, 2007).

Pese a que la FVT se reconoce como una unidad biogeográfica, su historia geológica y climática, y la distribución de sus especies, la hacen una de las provincias biogeográficas más heterogéneas y complejas del país (Espinosa y Ocegueda, 2007). Dicha complejidad ha sido identificada por diversos autores, quienes han detectado relaciones múltiples con otras provincias en porciones de su territorio (Corona et al., 2007; Navarro- Sigüenza et al., 2007; Flores-Villela y Canseco-Márquez, 2007), elevado número de nodos biogeográficos, producto de su condición transicional entre 2 reinos (Luna et al., 2000; Álvarez-Mondragón y Morrone, 2004; Escalante et al., 2004; Morrone y Gutiérrez, 2005; Corona et al., 2007; Morrone, 2010); así como inconsistencias en los criterios de delimitación y límites geográficos (Escalante et al., 2007) (Cuadro 1).

El hecho de que los mamíferos se encuentren distribuidos ampliamente en el territorio, estén bien representados en las colecciones (Escalante et al,. 2007), presenten gran diversidad y endemismos y exhiban intrincados patrones de distribución (Dice, 1943; Goldman y Moore, 1946; Ramí-
rez-Pulido y Castro-Campillo 1990, 1993; Fa y Morales 1991; Morrone y Escalante, 2002; Escalante et al., 2003, 2007), los hace un buen modelo para aproximarse a la historia biogeográfica de la FVT. Existen diversos trabajos que analizan la mastofauna de la FVT. Entre los enfocados al análisis de patrones de distribución, sobresalen los de Fa y Morales (1991) y Escalante et al. (2007). Fa y Morales (1991) realizaron la caracterización de la mastofauna y sus patrones de distribución; sin embargo lo hicieron a partir de los registros puntuales de recolección y un escenario geográfico que excede los límites de la provincia, un cuadrante geográfico ubicado entre los $17.5^{\circ}-21.5^{\circ} \mathrm{N}$ y $106^{\circ}-96.5^{\circ} \mathrm{O}$. Dicho cuadrante representa una área no natural ya que incluye porciones territoriales que forman parte de otras unidades biogeográficas (Simpson, 1964; Wilson, 1974; Pagel et al., 1991; Kaufman, 1995; Brown y Lomolino, 1998; Morrone, 2005). Así pues, la delimitación de Fa y Morales (1991) induce la inclusión de taxones característicos de otras provincias, cuyas distribuciones coinciden de manera marginal con la FVT, alterando el reconocimiento de patrones de la mastofauna en la provincia. Por ello, resulta necesario actualizar la información acerca de los patrones de distribución de los mamíferos de la FVT, a partir de una delimitación geográfica natural (Escalante et al., 2007).

Por su parte, a partir de un análisis de parsimonia de endemismos (PAE) de los registros de mamíferos en la FVT, Escalante et al. (2007) redefinieron los límites de la provincia mediante una cota altitudinal de 1000 $\mathrm{m}$, así como la lista de especies que conforman su mastofauna, al eliminar los elementos marginales. Estos mismos autores detectaron 2 unidades biogeográficas, este y oeste, las cuales sugirieron que fueran consideradas distrito biogeográfico (Fig. 1). Es importante mencionar que esta distinción biótica, entre la porción este y oeste de la provincia, ya había sido registrada por otros autores para diversos grupos taxonómicos (Cuadro 1), por lo que la caracterización de ambas porciones como subunidades parece necesaria para corroborar la naturalidad biogeográfica de la propuesta.

Dentro del ambiente físico se pueden encontrar varios factores que influyen en la riqueza de especies de un área determinada, entre los que se encuentran la latitud, la altitud, la temperatura, la diversidad de hábitat, entre otros (Patterson et al., 1989). Dado que las especies presentan una distribución espacial limitada, el número de especies se modifica con cambios en estas variables, lo que resulta en diferentes patrones geográficos (Illoldi, 1994; Peterson y Holt, 2003).

A lo largo de los últimos años se han desarrollado diversas aplicaciones geográficas, las cuales ofrecen nuevas posibilidades para el entendimiento de la diversidad 
Cuadro 1. Resumen de los distintos trabajos que analizan los patrones de la biodiversidad en la Faja Volcánica Transmexicana

\begin{tabular}{|c|c|c|c|}
\hline Citas & Taxones & Enfoquel metodología & $\begin{array}{c}\text { Elementos biogeográficos } \\
\text { identificados }\end{array}$ \\
\hline $\begin{array}{l}\text { Fa y } \\
\text { Morales, } \\
1991\end{array}$ & Mamíferos & $\begin{array}{l}\text { Revisión de la } \\
\text { diversidad, patrones } \\
\text { ecológicos análisis } \\
\text { complementariedad }\end{array}$ & $\begin{array}{l}\text { Patrón de diversidad de la } \\
\text { mastofauna detectando } 2 \text { áreas } \\
\text { de concentración de taxones, } \\
\text { una al O y otra en al SE. Patrón } \\
\text { de diversidad de este a oeste } \\
\text { en Rodentia, Lagomorpha y } \\
\text { Artiodactyla; distribución más } \\
\text { o menos homogénea en la parte } \\
\text { centro-este, para Carnivora e } \\
\text { Insectivora. Patrón de endemismo } \\
\text { asociado con la parte central del } \\
\text { polígono y las altitudes de } 2000 \text { - } \\
3000 \text { m. }\end{array}$ \\
\hline
\end{tabular}

Ferrari et

al.,2000

Luna et al.

2000

$\begin{array}{ll}\begin{array}{l}\text { Álvarez- } \\ \text { Mondragón } \\ \text { y Morrone, }\end{array} & \text { Aves } \\ 2004 & \\ \begin{array}{l}\text { Escalante et } \\ \text { al., } 2004\end{array} & \begin{array}{l}\text { Mamíferos } \\ \text { neárticos }\end{array}\end{array}$

Morrone y

Gutiérrez,

2005

Fuller et al., 2006
Mamíferos no voladores
Geológico

Panbiogeografía

(análisis de trazos con

PAE)

Panbiogeografía

(análisis de trazos)

Panbiogeografía

(análisis de trazos)

Panbiogeografía

(análisis de trazos)

Análisis de complementariedad y rareza

Evolución geológica progresiva, del Mioceno medio al Holoceno y de ONS a ESE, identificando 3 periodos: 1) Mioceno tardío (aprox. hace 13.8 Ma), afectando porciones este y centro; 2)

Plioceno inferior ( hace 5 Ma. aprox), afectando nuevamente porciones este $y$ centro; y 3) Plioceno tardíoCuaternario (aprox. $2 \mathrm{Ma}$ ), impactando principalmente el sector este del polígono.

Nueve trazos generalizados y 1 nodo en el $\mathrm{E}$.

Siete trazos generalizados, 1 nodo O (Jalisco), 1 nodo centro (Edo. Méx) y 1 nodo E (N Oaxaca).

Cuatro trazos generalizados, 3 nodos: 2 en centro, 1 en centro-E y 1 en $\mathrm{E}$

Veintidos trazos generalizados, 11 nodos en la porción centro-E, 1 en centro y 2 en centro-O.

Identificación de 3

unidades, de acuerdo con la composición de la fauna: $\mathrm{SO}, \mathrm{N}$-centro y E.

Los nodos encontrados son evidencia de un área bióticamente compleja, en la que se ha registrado una activa diversificación de los mamíferos.

Dado que la mayoría de los nodos en el país se encuentran en la FVT, 14 de 26 , ésta es una zona compleja y evolutivamente activa

Presencia de 99 especies. 
Cuadro 1. Continúa

Citas Taxones $\quad$ Enfoquel metodología $\quad \begin{gathered}\text { Elementos biogeográficos } \\ \text { identificados }\end{gathered}$ Interpretación biogeográfica

\begin{tabular}{lll}
\hline $\begin{array}{l}\text { Alcántara y } \\
\text { Paniagua, }\end{array}$ & $\begin{array}{l}\text { Plantas } \\
\text { endémicas }\end{array}$ & $\begin{array}{l}\text { Análisis de diversidad y } \\
\text { de complementariedad }\end{array}$ \\
&
\end{tabular}

Patrón de endemismo asociado con cota altitudinal de 1 500-2 500 m. Patrón de rareza coincidente con patrón de riqueza. Zonas con mayor riqueza de especies endémicas en la porción centro-E (10-18 taxones) y O (514 taxones).

$\begin{array}{lll}\begin{array}{l}\text { Contreras- } \\ \text { Medina et al., }\end{array} & \text { Gimnospermas } & \begin{array}{l}\text { Análisis de similitud } \\ \text { (UPGMA) }\end{array} \\ \text { Conona et al., } & \text { Coleoptera } & \begin{array}{l}\text { Panbiogeografía } \\ \text { (análisis de trazos con } \\ 2007\end{array} \\ & \text { PAE) }\end{array}$

Distribución de los taxones en 2 agrupaciones de áreas: centro y centro-E.

Panbiogeografía (PAE) Cladograma identificando 2

Revisión de la diversidad

y Canseco-

Anfibios y reptiles

Márquez,

2007

Lozano-

García y

Ceballos-

Ferriz, 2007

Navarro-

Aves

Panbiogeografía

Sigüenza et

al.,2007
(PAE)
Tres trazos generalizados. agrupaciones de áreas: E y O.

La porción NE de la FVT

(Puebla) se encuentra relacionada con la Sierra Madre Oriental, la SE con la Sierra Madre del Sur, la S-centro con la cuenca del Balsas, y la NO con la Sierra Madre Occidental y Costa del Pacífico mexicano.

Reconocimiento de 2 unidades biogeográficas mayores: $\mathrm{E}$ y $\mathrm{O}$.

Patrón de diversidad beta de herpetofauna de E-O; gradiente de riqueza de endemismos de reptiles deO-E; gran concentración de anfibios endémicos en SE de la provincia.

Gran parte del Cenozoico (previo a la formación de la FVT), climas más húmedos que los actuales; condiciones más frías durante la formación de la FVT, resultado de aumento en el intemperismo y la reducción en los niveles de $\mathrm{CO} 2$; y aproximadamente $3.5 \mathrm{Ma}$, cambio en el patrón de circulación atmosférica y mezcla de elementos bióticos neotropicales y neárticos.

Cladograma identificando 2 agrupaciones de áreas: E y $\mathrm{O} ; 2$ zonas de alta riqueza de especies: E (Veracruz) y O (Nayarit- Jalisco) y gradiente de endemismos de $\mathrm{E}$ a $\mathrm{O}$.
Riqueza y endemismos heterogéneamente distribuidos. La FVT no puede ser considerada como una provincia biótica natural.

Reconocimiento de 2 unidades biogeográficas mayores - E y Orelacionadas con la geomorfología, condiciones ecológicas actuales y zonas de contacto biótico al rededor. 
Cuadro 1. Continúa

\begin{tabular}{|c|c|c|c|c|}
\hline Citas & Taxones & Enfoque/ metodología & $\begin{array}{c}\text { Elementos biogeográficos } \\
\text { identificados }\end{array}$ & Interpretación biogeográfica \\
\hline $\begin{array}{l}\text { Toledo et } \\
\text { al.,2007 }\end{array}$ & $\begin{array}{l}\text { Cerambycidae } \\
\text { (Coleoptera) }\end{array}$ & $\begin{array}{l}\text { Panbiogeografía } \\
\text { (análisis de trazos) }\end{array}$ & $\begin{array}{l}\text { Dos trazos generalizados en } \\
\text { bosques montanos. }\end{array}$ & $\begin{array}{l}\text { Presencia de trazos, } \\
\text { soporta la hipótesis de } \\
\text { que es un área compleja } \\
\text { donde elementos bióticos } \\
\text { de distinto origen se } \\
\text { superponen. }\end{array}$ \\
\hline $\begin{array}{l}\text { Velasco de } \\
\text { León et al., } \\
2007\end{array}$ & - & Paleontológico & $\begin{array}{l}\text { Afinidad del registro en } 3 \text { bloques: } \\
\text { oriental (Pue- Tlax-Ver), central } \\
\text { (cuenca de México-EdoMex-Hgo- } \\
\text { Gto-Mich-Mor-Qro) y occidental } \\
\text { (Col-Jal-Nay). }\end{array}$ & $\begin{array}{l}\text { Identificación de } 3 \\
\text { principales agrupaciones de } \\
\text { áreas: E, centro y O. }\end{array}$ \\
\hline $\begin{array}{l}\text { Villaseñor y } \\
\text { Ortiz, } 2007\end{array}$ & Asteraceae & $\begin{array}{l}\text { Revisión de la } \\
\text { diversidad }\end{array}$ & $\begin{array}{l}\text { Patrón de endemismos } \\
\text { para Asteraceae, de O a E; } \\
\text { identificación de } 2 \text { áreas con gran } \\
\text { riqueza de endemismos, } 1 \text { en } \\
\text { Veracruz y } 1 \text { en Jalisco; patrón de } \\
\text { endemismos para musgos en el E. }\end{array}$ & $\begin{array}{l}\text { La riqueza y los } \\
\text { endemismos asociados } \\
\text { con la porción occidental, } \\
\text { geológicamente más } \\
\text { antigua. }\end{array}$ \\
\hline $\begin{array}{l}\text { García- } \\
\text { Marmolejo et } \\
\text { al., } 2008\end{array}$ & $\begin{array}{l}\text { Mamíferos } \\
\text { neotropicales }\end{array}$ & $\begin{array}{l}\text { Panbiogeografía } \\
\text { (análisis de trazos) }\end{array}$ & $\begin{array}{l}\text { Un nodo centro-O (Michoacán) y } \\
\text { otro en el E (Veracruz). }\end{array}$ & - \\
\hline $\begin{array}{l}\text { Escalante et } \\
\text { al., } 2009\end{array}$ & & $\begin{array}{l}\text { Análisis de } \\
\text { endemicidad (NDM) }\end{array}$ & $\begin{array}{l}\text { Identificación de } 2 \text { áreas de } \\
\text { endemismo que incluyen } \\
\text { territorios de la FVT, una en la } \\
\text { porción E y otra en la parte O de } \\
\text { la provincia. }\end{array}$ & $\begin{array}{l}\text { Dos diferentes áreas } \\
\text { asignadas a la categoría de } \\
\text { distrito. }\end{array}$ \\
\hline
\end{tabular}

biológica (Peterson et al. 2003). Tal es el caso de los Sistemas de Información Geográfica (SIG), que han hecho posible la construcción de mapas utilizando información geográfica precisa y relacionarlos con las áreas de riqueza de especies, prioridades de conservación, entre otras. De igual forma, los modelos de nicho ecológico o modelos de distribución potencial representan una herramienta útil en biogeografía, ecología y conservación (Peterson et al., 1999; Anderson et al., 2002), ya que brindan aproximaciones que combinan datos de la presencia de especies con variables ambientales (temperatura, precipitación, tipos de vegetación, elevación, entre otras) para crear modelos de distribución relacionados con estimadores ambientales (Edith y Leathwick, 2009). Este tipo de modelos permite conjuntar los factores que definen el hábitat, con el fin de realizar un análisis más completo sobre la distribución de las especies e, identificar, a partir de éste, los patrones de distribución (Illoldi et al., 2008).

En esta contribución se pretende identificar y actualizar, mediante el uso de un SIG y modelos de distribución potencial, la información acerca de los patrones biogeográficos (endemismo, riqueza latitudinal, riqueza altitudinal y de tipo de hábitat) de los mamíferos terrestres en la provincia de la FVT, dentro de los límites que sugieren Escalante et al. (2007). Asimismo, se caracterizan los distritos propuestos por estos autores; a partir de ello y de una revisión acerca de los trabajos biogeográficos realizados en la zona, se discute la validez de subdividir la FVT en 2 distritos.

\section{Materiales y métodos}

Para definir el área de estudio se eligió como mapa base la propuesta de la FVT de Escalante et al. (2007), desarrollada a partir de criterios morfotectónicos y mastofaunísticos, y se analizaron los patrones de distribución (riqueza y endemismos) de la mastofauna de la provincia. El universo taxonómico empleado fue la lista de especies identificadas por Escalante et al. (2007) como la mastofauna de la provincia, la cual incluye 152 especies y excluye los taxones con distribución marginal en la FVT.

El análisis de la distribución de los taxones se desarrolló a partir de modelos de distribución potencial de cada una de las especies; dichos modelos fueron generados a partir de de ejemplares de colecciones, de la literatura y 
de una base de datos con 19058 registros, compilada en el Laboratorio de Sistemas de Información Geográfica del Instituto de Biología, UNAM (Sánchez-Cordero et al., 2005), siguiendo la nomenclatura de Ramírez-Pulido et al. (2005). Además de lo antes mencionado, en la generación de los modelos se utilizaron mapas digitales de variables ambientales (elevación, pendiente, aspecto, parámetros climáticos y vegetación) y el modelador GARP (Genetic Algorithm for Rule-set Production) (Stockwell, 1999). Más detalles acerca de la metodología empleada pueden consultarse en Escalante et al. (2007).

Para identificar los patrones de la mastofauna, se contrastó el comportamiento espacial de los distintos modelos de distribución de las especies con información digitalizada de información geológica (Marín y TorresRuata, 1990), topográfica (modelo de digital de elevación, Conabio, 1997; www.conabio.gob.mx), climatológica (Conabio, 1998; www.conabio.gob.mx), uso de suelo y vegetación (Rzedowski y Reyna-Trujillo, 1990, www. conabio.gob.mx) y vegetación transformada (INEGI e IG, UNAM, 2000). La visualización y las operaciones entre los mapas se realizó utilizando el programa ArcView 3.2 (ESRI, 1999).

Para identificar los patrones de riqueza y endemismos, cada modelo de distribución potencial fue superpuesto al mapa base y a una cuadrícula de $0.5^{\circ}$ de latitud $x 0.5^{\circ}$ de longitud (70 cuadros) y se cuantificó la riqueza total (diversidad gama, de toda el área de estudio) y el número de especies por cada cuadro (diversidad alfa). Es importante aclarar que todos los cuadros fueron tratados igual, independientemente del área de intersección con el mapa base. Con el fin de resumir la información y facilitar su visualización, los cuadros fueron categorizados de acuerdo con el número de especies en 5 clases iguales de intervalos iguales; asimismo, se hizo una descripción de la composición de la mastofauna, para lo cual se cuantificó la representatividad de cada uno de los géneros en los valores de riqueza y endemismo.

Para describir la distribución de la riqueza de especies y de endemismos en términos de la altitud se superpuso el modelo de elevación al polígono de la FVT, previamente dividido en 8 intervalos cada $500 \mathrm{~m}$, con los modelos de distribución potencial de cada especie. Para cada intervalo, se calculó la riqueza de especies y endemismos y la representatividad numérica de éstas por orden.

Para el caso de la variable latitud, el mapa base de la FVT se dividió en 5 bandas, de los $18^{\circ} 30^{\prime}$ a los $21^{\circ} 00^{\prime}$, donde cada una de éstas abarca intervalos de $30^{\prime}$. Se calculó el área total y el número de especies coincidentes (riqueza absoluta) en las 5 bandas, así como la riqueza relativa - porcentaje de especies de acuerdo con el porcen- taje de área que ocupa cada banda- con el fin de evitar el efecto del tamaño del área sobre los valores obtenidos.

En el caso de la vegetación, el modelo de distribución de cada una de las especies fue superpuesto con el mapa digital de la vegetación y se cuantificó el número de especies con distribución coincidente en cada tipo de vegetación (i.e bosque de pino-encino), la proporción del área de distribución coincidente con cada tipo de vegetación dentro de la FVT, así como la proporción del área coincidente para cada orden.

Por otra parte, con el fin de corroborar la naturalidad de la propuesta de distritos de Escalante et al. (2007) (Fig. 1), se caracterizaron los terrenos de cada distrito, para lo cual se superpuso el mapa base y los polígonos de los distritos a mapas digitales con información geológica, topográfica, climática y de tipo de vegetación, así como los valores de riqueza y endemismo calculados en este trabajo, para hacer una detallada descripción de la estructura geográfica de las distintas variables sobre los terrenos de cada distrito. Finalmente se llevó a cabo una revisión de los trabajos biogeográficos en los que se analiza la distribución de las variables bióticas en la FVT, con el fin de contrastar los resultados aquí obtenidos.

En todos los casos, la visualización y análisis cartográfico se realizó utilizando Arc View 3.2 (ESRI, 1999).

\section{Resultados}

Se encontró que el 51\% (78) de las especies se distribuye en una de las 2 porciones, mientras que el 49\% (74) restante, en ambas. La porción oeste se encuentra definida por el $26.3 \%$ de las especies (40), de las cuales 5 son exclusivas de la provincia, mientras que la porción este por el $25 \%$ de las especies (38), 6 de ellas endémicas de la FVT (Apéndice 1).

Composición de la mastofauna. De las 152 especies que componen la mastofauna de la FVT, 49 exhiben distribuciones exclusivas a los límites geopolíticos de México y de éstas, 14 son endémicas a la FVT. El 76.1.\% de la riqueza está compuesto por roedores y murciélagos (44.1\% y 32\%, respectivamente). El orden Rodentia posee 67 especies, 34 exclusivas de México, de las cuales 11 son endémicas de la FVT; Chiroptera posee 49 especies, 5 de las cuales son exclusivas de México. Del resto de los órdenes presentes, Carnivora aporta el 9.9\% de la riqueza con 15 especies, ninguna endémica de la FVT; Soricomorpha aporta el 6.5\% de la riqueza con 10 especies, 7 de éstas exclusivas de México y 2 endémicas de la FVT; Lagomorpha representa el 3.2\% con 5 especies, 1 de éstas endemica de la FVT; Didelphimorpha, 2.6\% con 4 especies; y tanto Artiodactyla como Cingulata $0.6 \%$, con 1 especie cada uno (Fig. 2). 


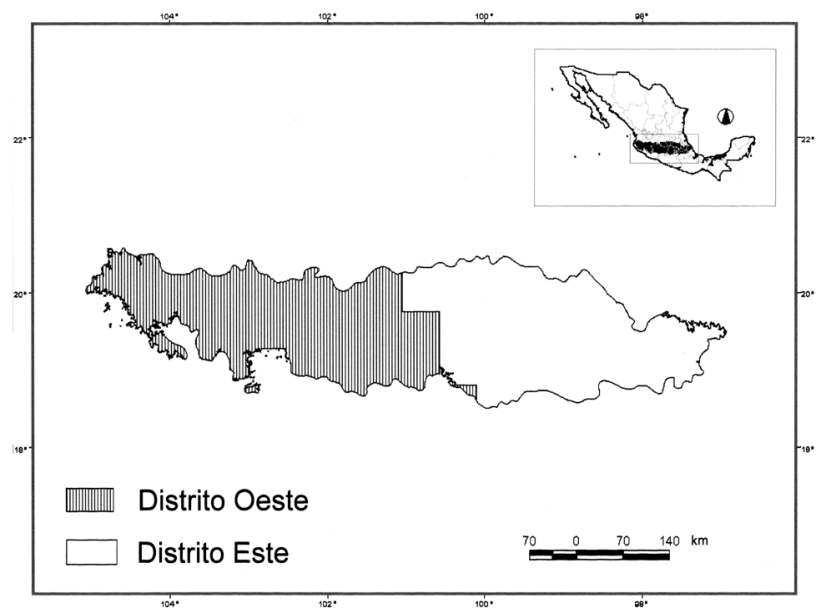

Figura 1. Propuesta de regionalización para la FVT de Escalante et al. (2007); se destacan los distritos este y oeste.

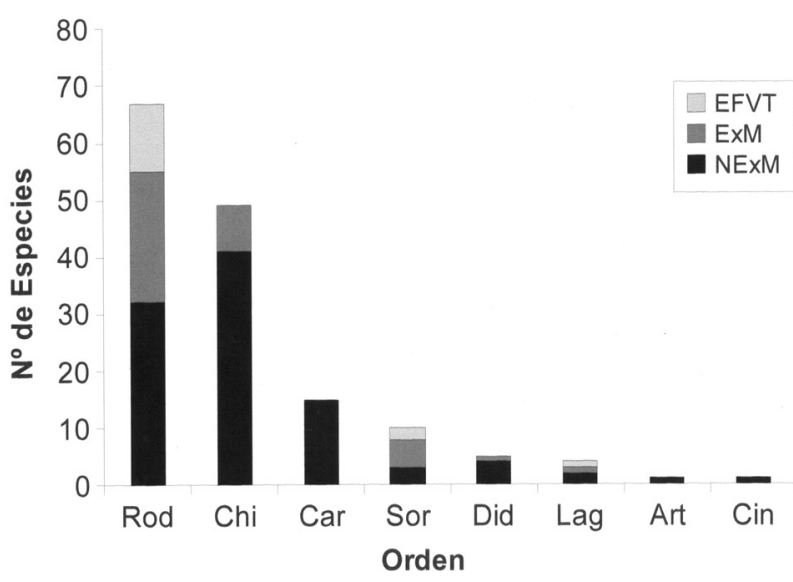

Figura 2. Diversidad de la mastofauna en la FVT correspondiente a cada orden. Rod, Rodentia; Chi, Chiroptera; Car, Carnivora; Sor, Soricomorpha; Lag, Lagomorpha; Art, Artiodactyla; Cin, Cingulata. EFVT, endémicas de la FVT; ExM, exclusivas de México; NexM, no exclusivas de México.

Riqueza. El promedio de especies por cuadro fue de 80.9. Se observó que la porción este exhibe un mayor número de especies, identificándose 3 principales agrupaciones de cuadros con máxima riqueza (101 a 116 especies), 2 en la porción este del polígono y 1 en la parte oeste. Acorde con esta distribución de mayor riqueza de especies en la porción este, la porción oeste cuenta con mayor número de cuadros con valores mínimos, en especial uno con 18 especies (Fig. 3).

Gradiente altitudinal. La riqueza de especies se concentró principalmente en 2 intervalos. De los 1500 y los $2500 \mathrm{~m}$, poco más del $50 \%$, principalmente roedores, y de los 1000 a los $1500 \mathrm{~m}$, principalmente por especies de murciélagos. Asimismo, se detectó que por encima de los $2500 \mathrm{~m} \mathrm{la}$ riqueza disminuye drásticamente.

Gradiente latitudinal. Las bandas latitudinales del polígono que limitan tanto al norte como al sur con otras provincias fueron las más ricas en número de especies, con 34 y 21 especies por unidad de área ( $1 \%$ del área de la FVT), mientras que las 3 bandas centrales presentan valores mucho menores, que van de 5.8 a 4.5 especies por unidad de área; sin embargo, no se pudo confirmar un gradiente latitudinal. Afinidad de hábitat. Se observaron marcadas diferencias en el número de especies presentes en los distintos tipos de vegetación: más del 98\% presentó áreas de distribución que coinciden con el bosque de pino-encino (150 especies) y/o con el bosque tropical caducifolio (146 especies), seguidas de bosque mesófilo de montaña (116), vegetación acuática (114), pastizal (112), matorral xerófilo (110), bosque espinoso (100), bosque tropical subcaducifolio (94) y bosque tropical perennifolio (65). Al analizar la proporción de área que ocupan los taxones en los distintos tipos de vegetación, el bosque de pino-encino representó, en promedio, el 53\% de las áreas de distribución potencial de los mamíferos presentes en la provincia; para 95 especies este tipo de bosque significó más del 50\% del área potencial de su distribución, 35 con más del $70 \%$ y 5 de las cuales restringen su distribución únicamente a este tipo de vegetación (Fig. 4). En segundo lugar se encuentra el bosque tropical caducifolio, que representa el $30 \%$ del área de distribución potencial para los mamíferos de la FVT. En este hábitat, 22 especies presentan más del $50 \%$ de sus áreas de distribución potencial, 7 de las cuales más del $70 \%$ y 1 se restringe a este hábitat. $\mathrm{Al}$ cuantificar el área que ocupan las distintas especies en los tipos de vegetación se observó la misma tendencia en todos los órdenes, en donde el bosque de pino-encino es el hábitat con mayor riqueza específica, representando el $70.7 \%$ del hábitat de Artiodactyla, el 68\% de Soricomorpha, 56.6\% de Carnivora, $53.9 \%$ de Cingulata, $53.6 \%$ de Rodentia, 50.2\% de Didelphimorpha, $48.6 \%$ de Chiroptera y $6.4 \%$ de Lagomorpha (Fig. 5).

Especies exclusivas de México. De los 13 géneros con distribución exclusiva en México (Ramírez-Pulido et al., 2005), 12 tienen presencia en la FVT. Del total de especies exclusivas de México, aproximadamente el 32\% (es decir, 54 especies pertenecientes a 6 órdenes, 9 familias y 32 géneros) presenta registros dentro de la FVT. El mayor número de especies se concentra en la parte este. No se observó ninguna tendencia latitudinal y en su mayoría (aprox. 70\%) se encuentran asociadas con altitudes que van de los 1500 a los $2500 \mathrm{~m}$ y presentan una clara afinidad por los bosques de pino-encino y el tropical caducifolio, ya que la gran mayoría (98.1\%) son elementos potencialmente presentes en estos tipos de vegetación. 


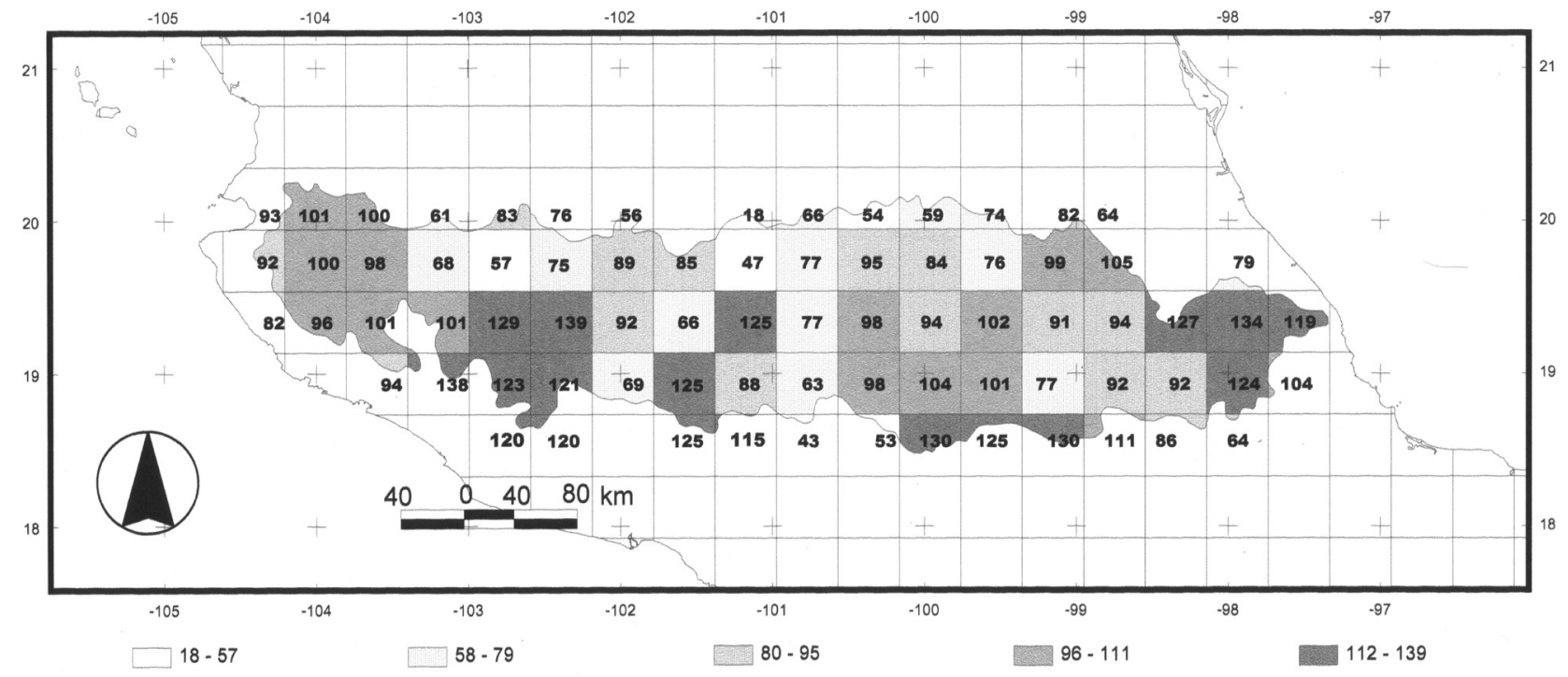

Figura 3. Número de especies por cuadro en la FVT. La riqueza de especies fue clasificada en 5 clases iguales.

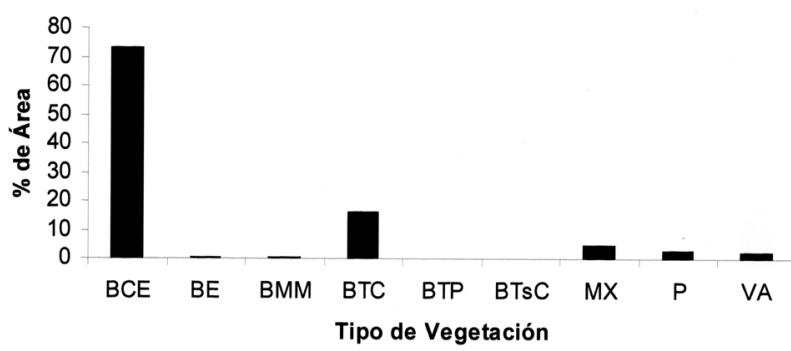

Figura 4. Proporción de área que ocupan las especies de mamíferos terrestres en los distintos tipos de vegetación dentro de la FVT. BCE, bosque de pino-encino; BE, bosque espinoso; BMM, bosque mesófilo de montaña; BTC, bosque tropical caducifolio; BTP, bosque tropical perenifolio; BTSc, bosque tropical subcaducifolio; MX, matorral xerófilo; P, pastizal; VA, vegetación acuática.

Especies endémicas de la FVT. Se identificaron 14 especies endémicas de la provincia, las cuales corresponden a 3 órdenes, 6 familias y 10 géneros. El grupo con mayor número de especies fue el de los roedores con 11, seguido de Soricomorpha,con 2, y Lagomorpha con 1 especie.

Riqueza de endemismos. De manera general, más o menos homogénea, 3 especies endémicas se encuentran ocupando la porción este de la provincia (Cratogeomys tylorhinus, Neotomodon alstoni y Reithrodontomys chrysopsis; 6 ocupan la porción este (Cratogeomys merriami, Peromyscus bullatus, Romerolagus diazi, Sorex macrodon, S. ventralis y Spermophilus perotensis), mientras que en la parte oeste del polígono se registran las 5 restantes (Cratogeomys gymnurus, Liomys spectabilis, Nelsonia goldmani, Reithro-

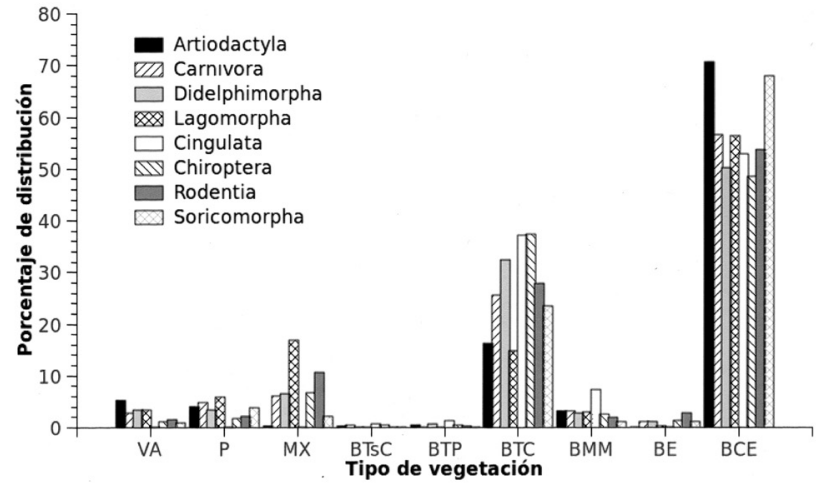

Figura 5. Porcentaje de área ocupada por las especies correspondientes a cada orden, de acuerdo con los distintos tipos de vegetación según Rzedowski (1997). BCE, bosque de pinoencino; BE, bosque espinoso; BMM, bosque mesófilo de montaña; BTC, bosque tropical caducifolio; BTP, bosque tropical perennifolio; $\mathrm{Bts} C$, bosque tropical subcaducifolio; $\mathrm{MX}$, matorral xerófilo; P, pastizal; VA, vegetación acuática.

dontomys hirsutus y Zygogeomys trichopus). Sin embargo, dada la posición, forma y extensión de sus áreas de distribución, prácticamente muestran un patrón sin superposición entre sí (alopátrida).

Gradiente altitudinal de endemismos. El intervalo de 2000 a $3000 \mathrm{~m}$, conforma casi el 70\% del área total ocupada por estas especies. Este intervalo comprende más del 75\% del área de distribución de 7 de las 14 especies endémicas (Cratogeomys merriami, Neotomodon alstoni, Reithrodontomys chrysopsis, Romerolagus diazi, Sorex macrodon, Spermophilus perotensis y Zygogeomys trichopus) y el 
$100 \%$ de 2 de ellas (Nelsonia goldmani y Peromyscus bullatus). En cuanto al intervalo altitudinal que va de 1000 a $2000 \mathrm{~m}$ representa el $25 \%$ del área total de la distribución de las especies endémicas de la FVT, porcentaje que equivale a más del $70 \%$ sus áreas de distribución en 3 de éstas (Cratogeomys gymnurus, Liomys spectabilis y Reithrodontomys hirsutus). En las 2 restantes (Cratogeomys tylorhinus y Sorex ventralis), sus distribuciones se extienden de manera intermedia a los intervalos altitudinales de $2000-3000$ y $1000-2000 \mathrm{~m}$.

Gradiente latitudinal de endemismos. A pesar de que no se observó un gradiente latitudinal en la distribución de estos taxones, estos se encontraron preferentemente ocupando la parte media de la provincia, principalmente en las zonas montañosas.

Afinidad de hábitat en endemismos. Con excepción del bosque tropical perennifolio y el bosque tropical subcaducifolio, la mayoría de las especies endémicas de la FVT presentaron intersecciones de sus áreas de distribución con los principales tipos de vegetación descritos previamente. Sin embargo, al comparar la representatividad de cada una de estas comunidades sobre el área total ocupada por las especies endémicas, se observa una clara dominancia del bosque de pino-encino al representar más del $73 \%$ del área ocupada por las especies. De acuerdo con los modelos de distribución, 3 de ellas se restringen a este tipo de vegetación (Nelsonia goldmani, Neotomodon alstoni y Zygogeomys trichopus) (Fig. 6). En segundo lugar, y muy por debajo del bosque de pino-encino, se encuentra el bosque tropical caducifolio, representando el 16\% del área total ocupada por las especies endémicas de la FVT. Esta vegetación resulta de gran importancia para Reithrodontomys hirsutus, ya que conforma el $79 \%$ del área de distribución potencial. Aunque con un porcentaje total bajo (4.6\%), el matorral xerófilo resultó de gran importancia para Peromyscus bullatus, ya que conforma casi el $60 \%$ del área de distribución potencial (Fig.6).

Caracterización de los distritos. Al comparar el comportamiento espacial de la información geológica, altitudinal, climática y de tipo de hábitat, con las unidades biogeográficas reconocidas por Escalante et al. (2007), se observó una clara diferencia entre la porción este y oeste de la provincia. De acuerdo con la información climática, en la porción este dominan climas tipo $\mathrm{C}\left(\mathrm{w}_{1}\right)$ y $\mathrm{C}\left(\mathrm{w}_{2}\right)$ (templados subhúmedos), $\mathrm{BS}_{1} \mathrm{kw}$ (semiárido templado) y $\mathrm{Cb}^{\prime}\left(\mathrm{w}_{2}\right)$ (semifrío subhúmedo), mientras que en la porción oeste dominan climas $(\mathrm{A}) \mathrm{C}\left(\mathrm{w}_{\mathrm{o}}\right)$ (semicálido subhúmedo) (CONABIO, 1998). Lo mismo ocurre con la distribución de la vegetación al interior de cada porción: en la del oeste el bosque tropical caducifolio representa el $49 \%$ del área, mientras que en la este representa el $8 \%$, dominada por el bosque de pino-encino $(57 \%)$ y el matorral xerófilo $(21 \%)$. Se obser-

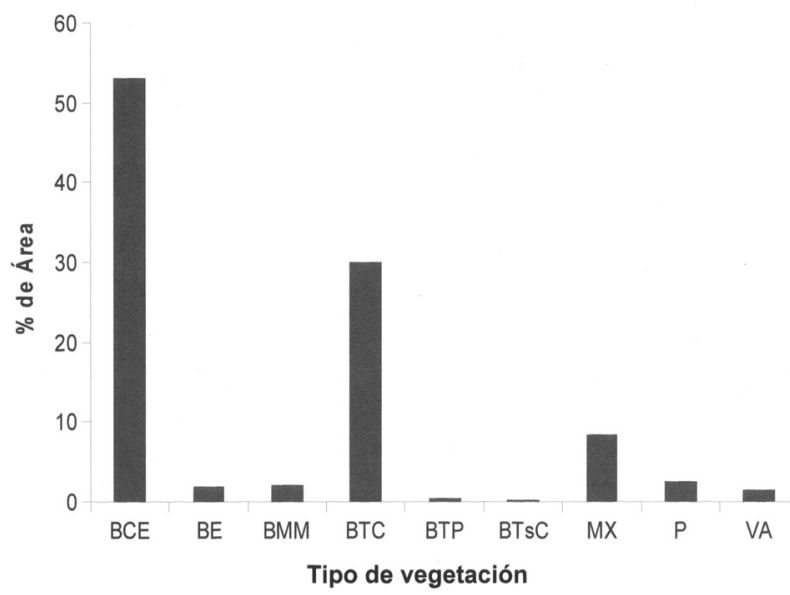

Figura 6. Porcentaje de área que representan los distintos tipos de vegetación en la distribución de las especies endémicas de la FVT. BCE, bosque de pino-encino; BE, bosque espinoso; BMM, bosque mesófilo de montaña; BTC, bosque tropical caducifolio; BTP, bosque tropical perennifolio; BtsC, bosque tropical subcaducifolio; MX, matorral xerófilo; P, pastizal; VA, vegetación acuática.

varon marcadas diferencias en el gradiente altitudinal entre ambas porciones: en la porción este predominan altitudes que van de los 2000 a los $3000 \mathrm{~m}$ (73\% del área), mientras que en la oeste dominan altitudes menores a los $2000 \mathrm{~m}$ (71\% del área).

En términos geológicos, el margen este del polígono posee rocas jurásicas, mientras que el oeste presenta rocas intrusivas del Cenozoico, Mesozoico y Paleozoico, en tanto que en la FVT en general predominan elementos volcánicos del Cenozoico superior y del Pleistoceno. En el centro-sur y oeste también existen pequeños manchones de Mesozoico, Paleozoico y Precámbrico, y amplias extensiones de Cenozoico medio volcánico (Marín y Torres-Ruata, 1990).

\section{Discusión}

La provincia de la FVT es una zona rica en especies de mamíferos y con alto número de endemismos, que además posee representantes de casi todos los géneros exclusivos de México. Sus altos valores de diversidad alfa (80.9 especies por cuadro) y gama (152 especies) se relacionarían con 4 causas, que de manera conjunta podrían promover valores altos de diversidad beta (Arita, 1997): la compleja historia geológica, la gran heterogeneidad topográfica y ambiental, la condición biótica transicional del área y procesos recientes de especiación (neoendemismos).

De las 152 especies de mamíferos que conforman la mastofauna de la FVT, roedores y murciélagos son los 
principales representantes al conformar el $76.1 \%$ de las especies. La riqueza de especies se encuentra distribuida de manera no homogénea entre la porción este y oeste de la provincia, siendo la porción este la que posee mayor número de especies. Dicha riqueza se encuentra asociada con 2 intervalos altitudinales, el que va de 1000 a 1500 $\mathrm{m}$, dominada por especies de murciélagos y el de 1500 a $2500 \mathrm{~m}$, en el cual se distribuye la mayoría de las especies de roedores; en tanto que los terrenos por encima de los 2 $500 \mathrm{~m}$ exhiben baja diversidad. En cuanto a la distribución de los endemismos, predominantemente entre los $2000 \mathrm{y}$ los $3000 \mathrm{~m}$, consideramos que en gran medida es resultado del aislamiento geográfico que ofrecen los distintos picos montañosos. Lo anterior podría deberse al reciente origen, tanto de los principales picos montañosos, como de la mayoría de las especies endémicas (Fa y Morales, 1991).

El bosque de pino-encino y el bosque tropical caducifolio son los tipos de vegetación sobre los cuales se extiende el $83 \%$ del área de distribución de los mamíferos, esto podría relacionarse con 3 elementos; por un lado, la abundancia de estos tipos de vegetación en la provincia, ocupando respectivamente el $53 \%$ y $26 \%$ del área, lo cual a su vez se encuentra fuertemente influenciado por factores fisiográficos y climáticos; la marcada afinidad de los roedores por el bosque de pino-encino, determinada por los hábitos alimenticios; la preferencia del orden por altitudes mayores de los $2000 \mathrm{~m}$ (Ramírez-Pulido et al., 2005; Rodríguez et al., 2003) y el efecto enmascarado de las restricciones altitudinales de la distribución de los murciélagos, para los que la distribución se encuentra más relacionada con la altitud y no tanto con el tipo de vegetación (Schmidly, 1977; Arita, 1993). Estos resultados contrastan con los de Fa y Morales (1991), quienes en orden de importancia identifican el bosque mesófilo, bosque tropical perennifolio y el bosque de pino-encino como los tipos de vegetación en los cuales se desarrolla la mastofauna de la FVT.

En cuanto a un patrón latitudinal, los resultados de este estudio no corroboran lo registrado a escala continental (Wilson, 1974; McCoy y Connor, 1980), nacional (Arita, 1993) ni provincial (Fa y Morales, 1991), en que la riqueza disminuye gradualmente conforme aumenta la latitud. El desacuerdo con ese patrón quizás tiene que ver con que a esta escala, en la que dimensión y amplitud latitudinal son marcadamente menores que a escala nacional, su detección está siendo enmascarada. Por otra parte, la disminución en el número de especies en las latitudes medias de la FVT, pudiera relacionarse con el patrón altitudinal y el efecto de éste sobre el clima y la vegetación; ya que las principales formaciones montañosas, ubicadas precisamente en dichas latitudes, pudieran estar funcionando como barrera para las especies de afinidad neotropical y en menor grado para las neárticas. Un claro ejemplo de lo anterior es la distribución que exhiben las especies de quirópteros, evitando en gran parte las zonas montanas.

Los resultados obtenidos en este trabajo difieren en gran medida de los obtenidos en trabajos previos que analizan los patrones de distribución de la mastofauna en la FVT (Fa y Morales, 1991; Arita y Rodríguez, 2002; Fuller et al., 2006), debido a que los límites geográficos y taxones de mamíferos empleados no son equivalentes, lo que en gran parte puede deberse a que en dichos trabajos el universo de análisis fue definido a partir de una delimitación geográfica no natural de la FVT, y no tanto a cambios en la taxonomía.

Para Fa y Morales (1991), el área de estudio se sitúa entre $\operatorname{los} 17.5^{\circ}-21.5^{\circ} \mathrm{N}$ y $106^{\circ}-96.5^{\circ} \mathrm{O}$, incluye porciones costeras de Nayarit, Jalisco y Veracruz y abarca una extensión aproximada de $390000 \mathrm{~km}^{2}$. Arita y Rodríguez (2002) analizaron el área correspondiente al cuadrante "neártico-transicional" que ocupa porciones de las provincias de la cuenca del Balsas y FVT, incluyendo parte de las costas de Guerrero y Michoacán y abarca un área aproximada de $160000 \mathrm{~km}^{2}$. Finalmente, Fuller et al. (2006) definieron la lista de especies en torno a un polígono de la FVT, no especificando qué cartografía utilizaron ni cuáles fueron los límites geográficos específicos del polígono y los criterios utilizados para generarlo. En distinto grado, todos estos trabajos incluyen porciones costeras, así como porciones de la cuenca del Balsas, las cuales se diferencian de los terrenos de la FVT en términos de su geología, tipos de vegetación, clima y patrón altitudinal (Escalante et al., 2007). El hecho que estos trabajos incluyan estas porciones, donde la integración de elementos neotropicales es mayor, tiene como resultado la incorporación de taxones de Primates o Cingulata, o bien, de taxones con distribución marginal (únicamente en unas cuantas localidades) en la FVT. Los límites y lista de taxones empleados son resultado de criterios históricos (integridad fisiográfica y geológica), ecológicos (integridad climática, altitudinal y de tipo de hábitat) y eliminación de porciones en los que la mastofauna se integra por especies con distribución marginal a la provincia; es decir, sus áreas de distribución apenas coinciden con porciones limítrofes del territorio. Por otra parte, las delimitaciones geográficas de los trabajos mencionados no aclaran los criterios empleados en la formulación de los límites, incluyen porciones de territorio pertenecientes a otras provincias (i.e Balsas); en algunos casos incluyen porciones de océano en su territorio (i.e Fa y Morales, 1991 y Arita y Rodríguez, 2002 ); son más bien una delimitación geométrica y no biótica y en los hechos, aceptan que el componente biótico que define a la zona como provincia biogeográfica se encuentra conformado por criterios geométricos y no tanto por interacción 
histórica entre los componentes bióticos y ambientales (geología, clima, topografía, entre otros). Por ello, se considera que los patrones mastofaunísticos aquí descritos, identificados a partir de la delimitación geográfica y taxonómica de Escalante et al. (2007), son una aproximación robusta de los patrones biogeográficos de la mastofauna de la FVT.

De acuerdo con los patrones de distribución observados -abundancia de roedores asociados a bosque de pino-encino, altitudes por arriba de los $2000 \mathrm{~m} \mathrm{snm}$ y murciélagos limitando gran parte de su distribución a terrenos por debajo de los $1500 \mathrm{~m} \mathrm{snm}$ - la altitud y la vegetación son las variables que explican mejor la distribución de los mamíferos dentro de la FVT. Ambos patrones podrían estar relacionados con 3 fenómenos: la marcada afinidad de los roedores por el bosque de pino-encino, relacionada a su vez con sus hábitos alimenticios (Fa y Morales, 1991; Rodríguez et al., 2003; Ramírez-Pulido et al., 2005); la correlación de la vegetación con el patrón altitudinal y las restricciones ecológicas de los murciélagos, que raramente sobrepasan los $1500 \mathrm{~m}$ de altitud, donde la relación altitud-temperatura-humedad posiblemente funciona como barrera distribucional (Schmidly, 1977; Arita, 1993). Estos resultados corroboran lo que registraron $\mathrm{Fa}$ y Morales (1998) y Escalante et al. (2007), quienes señalaron la relevancia de estas 2 variables en la conformación de los patrones de distribución de los mamíferos y en la definición de los límites de la provincia como unidad mastofaunística, respectivamente.

La distribución geográfica de la mastofauna, tanto en riqueza y endemismos como en la conformación espacial de la geología, clima, topografía y vegetación, exhibe diferencias entre las porciones este y oeste, lo cual da sustento a la identificación de ambas porciones como distritos biogeográficos. La identificación de dichos distritos puede resumirse en los siguientes puntos: 1), riqueza de especies en 2 áreas, una al este y otra al oeste; 2), distribución de los taxones endémicos en 3 bloques, uno al este, otro al oeste y un tercero conformado por 3 especies con amplia distribución en la provincia; 3), clima, en su gran mayoría, templado subhúmedo y semifrío subhúmedo en la porción este y semicálido subhúmedo dominando el oeste; 4), geología de la parte este y centro de la provincia, resultado de procesos ocurridos durante el Mioceno tardío (aprox. hace $11.8 \mathrm{Ma}$ ) y Plioceno inferior (aprox. hace $5 \mathrm{Ma}$ ) y en el Plioceno tardío-Cuaternario (aprox. $2 \mathrm{Ma}$ ) en la oeste; 5), predominancia de altitudes entre los 2000 y los 3000 $\mathrm{m}$ en la porción este, y menores a los 2000 en el oeste y 6), comunidades dominantes de pino-encino y matorral xerófilo, ocupando respectivamente el $57 \%$ y $21 \%$ del área este, mientras que en el oeste, cubriendo el $49 \%$, domina el bosque tropical caducifolio.
El reconocimiento de los distritos este y oeste, con base en el patrón de diferenciación entre la porción este y oeste de la provincia, confirma los registros para mamíferos (Escalante et al., 2007, 2009), aves (Navarro-Sigüenza et al., 2007), gimnospermas (Contreras-Medina et al., 2007), anfibios y reptiles (Flores-Villela y Canseco-Márquez, 2007), Asteraceae (Villaseñor y Ortiz, 2007) y el registro paleontológico (Velasco de León et al., 2007).

La coincidencia entre la distribución de la biota, el reciente origen de algunas de las especies endémicas ( $\mathrm{Fa}$ y Morales, 1991) y la asincronía del origen geológico del territorio pudieran ser evidencia de que muchos de los patrones de homología biogeográfica presentes en la FVT se conformaron a partir de los últimos $11.8 \mathrm{Ma}$ y se consolidaron de manera más intensa en los últimos $3 \mathrm{Ma}$, producto de procesos geológicos, climáticos y bióticos. Estos elementos sugieren que cada una de estas porciones, donde se da la mayor integración histórica y ecológica en la provincia, se encuentra ligada con un componente biótico diferente, con una historia biogeográfica que las distingue, por lo que la propuesta de Escalante et al. (2007) de elevar a distrito cada una de estas porciones, parece lo más indicado.

\section{Agradecimientos}

A la Universidad Nacional Autónoma de México, donde se realizó con pleno apoyo y libertad este trabajo, $\mathrm{y}$ al financiamiento del proyecto 80370 de CONACYT. Al Dr. Víctor Sánchez-Cordero, entonces Jefe del Laboratorio de Sistemas de Información Geográfica del Instituto de Biología, UNAM y hoy director del propio Instituto, por permitirnos realizar este trabajo en las instalaciones del laboratorio.

\section{Literatura citada}

Alcántara, O. y M. Paniagua. 2007. Patrones de distribución y conservación de las plantas endémicas. In Biodiversidad de la Faja Volcánica Transmexicana, I. Luna, J. J. Morrone y D. Espinosa (eds.). Universidad Nacional Autónoma de México, México, D. F. p. 421-438.

Álvarez-Mondragón, E. y J. J. Morrone. 2004. Propuesta de áreas para la conservación de aves de México, empleando herramientas panbiogeográficas e índices de complementariedad. Interciencia 29:112-120.

Arita, H. T. y P. Rodríguez. 2002. Geographic range, turnover rate and the scaling of species diversity. Ecography 25:541-553.

Arita, H. T. 1993. Riqueza de especies de la mastofauna de México. In Avances en el estudio de los mamíferos de México, vol. 1, R. A. Medellín y G. Ceballos (eds.). Publicaciones Especiales. Asociación Mexicana de Mastozoología, México, 
D. F. p. 109-128.

Arita, H. T. 1997. Species composition and morphological structure of the bat fauna of Yucatan, Mexico. Journal of Animal Ecology 66:83-97.

Bradbury, J. P. 1997. Sources of glacial moisture in Mesoamerica. Quaternary International 43:97-110.

Brown, J. H. y M. V. Lomolino. 1998. Biogeography 2a. ed., Sinauer Associates, Inc. Pub. Sunderland. 691 p.

Challenger, A. 1998. Utilización y conservación de los ecosistemas terrestres de México: Pasado, presente y futuro. Conabio/ Instituto de Biología, UNAM/ Agrupación Sierra Madre, México, D. F. 847 p.

Conabio (Comisión Nacional para el Conocimiento y Uso de la Biodiversidad). 1997. Modelo digital del terreno. Escala 1:250000. México, D. F. www.conabio.gob.mx; última consulta: 15.I.2011.

Contreras-Medina, R., D. Castañeda-Aguado, A. GonzálezZamora. 2007. Gimnospermas. In Biodiversidad de la Faja Volcánica Transmexicana, I. Luna, J. J. Morrone y D. Espinosa (eds.). Universidad Nacional Autónoma de México. México, D. F. p. 129-138.

Corona, A. M, V. H. Toledo y J. J. Morrone. 2007. Análisis panbiogeográfico de especies de Buprestidae y Cerambycidae (Insecta: Coleoptera). In Biodiversidad de la Faja Volcánica Transmexicana, I. Luna, J. J. Morrone y D. Espinosa (eds.). Universidad Nacional Autónoma de México, México, D. F. p. 439-448.

Dice, L. R. 1943. The biotic provinces of North America. University of Michigan Press, Ann Arbor. p. 1-78.

Escalante, T. 2009. Un ensayo sobre regionalización biogeográfica. Revista Mexicana de Biodiversidad 80:551560.

Escalante, T., D. Espinosa y J. J. Morrone. 2003. Using parsimony analysis of endemicity to analyze the distribution of Mexican land mammals. The Southwestern Naturalist 48:563-578.

Escalante, T., G. Rodríguez, N. Gámez, L. León-Paniagua, O. Barrera y V. Sánchez-Cordero. 2007. Biogeografía y conservación de los mamíferos. In Biodiversidad de la Faja Volcánica Transmexicana, I. Luna, J. J. Morrone y D. Espinosa (eds.). Universidad Nacional Autónoma de México, México, D. F. p. 485-502.

Escalante, T., G. Rodríguez y J. J. Morrone. 2004. The diversification of Nearctic mammals in the Mexican Transition Zone. Biological Journal of the Linnaean Society 83:327-339.

Escalante, T., C. Szumik y J. J. Morrone. 2009. Areas of endemism of Mexican mammals: Re-analysis applying the optimality criterion. Biological Journal of the Linnean Society 98:468478.

Espinosa, D. y S. Ocegueda, 2007. Introducción. In Biodiversidad de la Faja Volcánica Transmexicana, I. Luna, J. J. Morrone y D. Espinosa (eds.). Universidad Nacional Autónoma de
México, México, D. F. p. 5-6.

ESRI. 1999. Arc View GIS 3.2. Environmental Systems Research Institute, Redlands, California.

Fa, J. E. y L. M. Morales. 1991. Mammals and protected areas in the Trans-Mexican Neovolcanic Belt. In Latin American mammalogy: history, diversity and conservation, M. Y . Mares y D. J. Schmidly (eds.). University of Oklahoma Press, Norman. p. 199-226.

Fa, J. E. y L. M. Morales. 1998. Patrones de diversidad de mamíferos de México. In Diversidad biológica de México: orígenes y distribución, T. P. Ramamoorthy, R. Bye, A. Lot y J. Fa (eds.). Instituto de Biología, UNAM, México, D. F. p. 315-352.

Ferrari, L., S. Conticelli, C. Vaggelli, C. Petrone y P. Manetti. 2000. Late Miocene mafic volcanism and intra-arc tectonics during the early development of the Trans-Mexican Volcanic Belt. Tectonophysics 318:161-185.

Ferrusquía-Villafranca. 2007. Ensayo sobre la caracterización y significación biológica. In Biodiversidad de la Faja Volcánica Transmexicana, I. Luna, J. J. Morrone y D. Espinosa (eds.). Universidad Nacional Autónoma de México, México, D. F. p. 7-24.

Flores-Villela, O. y L. Canseco-Márquez. 2007. Riqueza de la herpetofauna. In Biodiversidad de la Faja Volcánica Transmexicana, I. Luna, J. J. Morrone y D. Espinosa (eds.). Universidad Nacional Autónoma de México, México, D. F. p. 407-420.

Fuller, T., M. Munguía, M. Mayfield, V. Sánchez-Cordero y S. Sarkar. 2006. Incorporating connectivity into conservation planning: A multi-criteria case study from central Mexico. Biological Conservation 133:131-142.

García, E. 1988. Climas (clasificación de Koppen, modificado por García). Escala 1:1000 000. Comisión Nacional para el Conocimiento y Uso de la Biodiversidad, México, D.F. www.conabio .gob; última consulta: 15.I.2011.

García-Marmolejo, G., T. Escalante y J. J. Morrone. 2008. Establecimiento de prioridades para la conservación de mamíferos terrestres neotropicales de México. Mastozoología Neotropical 15:41-65.

Goldman, E. A y R. T. Moore. 1946. Biotic provinces of México. Journal of Mammalogy 26:347-360.

Halffter, G. 1976. Distribución de los insectos en la Zona de Transición Mexicana: Relaciones con la entomofauna de Norteamérica. Folia Entomológica Mexicana 35:1-64.

INEGI e IG (Instituto Nacional de Estadística y Geografía e Instituto de Geografía, UNAM). 2000. Inventario Forestal Nacional., Secretaría de Medio Ambiente y Recursos Naturales, México, D. F.

Kaufman, D. M. 1995. Diversity of New World mammals: diversity of latitudinal gradients of species and bauplans. Journal of Mammalogy 76:322-334.

Lozano-García, M. S. y S. Ceballos-Ferriz. 2007. Historia 
de la vegetación en el centro de México: Evidencias paleobotánicas. In Biodiversidad de la Faja Volcánica Transmexicana, I. Luna, J. J. Morrone y D. Espinosa (eds.). Universidad Nacional Autónoma de México, México, D. F. p. 273-288.

Luna, I., O. Alcántara, J. J. Morrone y D. Espinosa. 2000. Track analysis and conservation priorities in the cloud forests of Hidalgo, México. Diversity and Distributions 6:137-143.

Marín, C. S. y C. Torres-Ruata. 1990. Hidrogeología, mapa IV. 6. 3, escala 1: 4000 000. Atlas Nacional de México, vol. 2 Instituto de Geografía, UNAM, México, D. F.

McCoy, E. D. y E. F. Connor.1980. Latitudinal gradients in the species diversity of North American mammals. Evolution 34:193-203.

Morrone, J. J. 2001. Homology, biogeography and areas of endemism. Diversity and Distributions 7:297-300.

Morrone, J. J. 2004. La zona de transición sudamericana: Caracterización y relevancia evolutiva. Acta Entomologica Chilena 28:41-50.

Morrone, J. J. 2005. Hacia una síntesis biogeográfica de México. Revista Mexicana de Biodiversidad 76:207-252.

Morrone, J. J. 2009. Evolutionary biogeography: An integrative approach with case studies. Columbia University Press, New York. 301 p.

Morrone, J. J. 2010. Fundamental biogeographic patterns across the Mexican Transition Zone: An evolutionary approach. Ecography 33:355-361.

Morrone, J. J. y T. Escalante. 2002. Parsimony analysis of endemicity (PAE) of Mexican terrestrial mammals at different area units: when size matters. Journal of Biogeography 298:1095-1104.

Morrone, J. J. y A. Gutiérrez. 2005. Do fleas (Insecta: Siphonaptera) parallel their mammal host diversification in the Mexican Transition Zone? Journal of Biogeography 32:1315-1325.

Navarro-Sigüenza, A. G., A. Lira-Noriega, A. T. Peterson, A. Oliveras-de Ita y A. Gordillo-Martínez. 2007. Diversidad, endemismo y conservación de las aves. In Biodiversidad de la Faja Volcánica Transmexicana, I. Luna, J. J. Morrone y D. Espinosa (eds.). Universidad Nacional Autónoma de México, México, D. F. p. 461-484.

Pagel, M. D., R. M. May y A. R. Collie. 1991. Ecological aspects of the geographical distribution and diversity of mammalian species. American Naturalist 137:791-815.

Ramamoorthy, T. P., R. Bye, A. Lot y J. Fa. (eds.). 1998. Diversidad biológica de México: orígenes y distribución. Instituto de Biología, UNAM, México, D. F.

Ramírez-Pulido, J. y A. Castro-Campillo. 1990. Provincias mastofaunísticas, mapa IV.8.8A, escala 1: 4,000 000. Atlas Nacional de México, vol. 2. Instituto de Geografía, UNAM, México, D. F.

Ramírez-Pulido, J. y A. Castro-Campillo. 1993. Diversidad mastozoológica en México. Revista de la Sociedad Mexicana de Historia Natural 44:413-427.

Ramírez-Pulido J., J. Arroyo-Cabrales y A. Castro-Campillo. 2005. Estado actual y relación nomenclatural de los mamíferos terrestres de México. Acta Zoológica Mexicana (nueva serie) 91:21-82.

Rodríguez, P., J. Soberón y H. T. Arita. 2003. El componente beta de la diversidad de mamíferos de México. Acta Zoológica Mexicana (nueva serie) 89:241-259.

Rzedowski, J. y T. Reyna-Trujillo. 1990. Tópicos biogeográficos, mapa IV.8.3. Atlas Nacional de México, vol. 3. Instituto de Geografía, UNAM, México, D.F.

Schmidly, D. J. 1977. The mammals of Trans-Pecos Texas. Texas A\&M University Press, College Station. 225 p.

Simpson, G. 1964. Species density of North American recent mammals. Systematic Zoology 13:57-73.

Smith, H. 1941. Las provincias bióticas de México, según la distribución geográfica de las lagartijas del género Sceloporus. Anales de la Escuela Nacional de Ciencias Biológicas 2:103-110.

Toledo, V. H., A. M. Corona y J. J. Morrone. 2007. Track analysis of the Mexican species of Cerambycidae (Insecta: Coleoptera). Revista Brasileira de Entomologia 51:131-137.

Velasco de León, P., J. Arellano, A. Silva-Pineda y S. Yussim. 2007. Aspectos geológicos y paleontológicos. In Biodiversidad de la Faja Volcánica Transmexicana, I. Luna, J. J. Morrone y D. Espinosa (eds.). Universidad Nacional Autónoma de México, México, D. F. p. 25-38.

Villaseñor J. L. y E. Ortiz. 2007. La familia Asteraceae. In Biodiversidad de la Faja Volcánica Transmexicana, I. Luna, J. J. Morrone y D. Espinosa (eds.). Universidad Nacional Autónoma de México, México, D. F. p. 289-310.

Wilson, M. V. 1974. Analytical zoogeography of North American mammals. Evolution 28:124-140.v 
Apéndice 1. Especies de mamíferos potencialmente presentes en la Faja Volcánica Transmexicana. Los superíndices ${ }^{\mathrm{abc}}$ se refieren a su endemicidad. ${ }^{a}$ endémica de la FVT; bendémica del distrito este $\mathrm{y}^{\mathrm{c}}$ endémica del distrito oeste.

Anoura geoffroyi

Antrozous pallidus

Artibeus hirsutus

A. jamaicensis

Baeodon alleni

Baiomys musculus

B. taylori

Bassariscus astutus

Canis latrans

Carollia perspicillata

Chaetodipus hispidus

Chiroderma salvini

Choeronycteris mexicana

Conepatus leuconotus

Corynorhinus mexicanus

Cratogeomys fumosus

C. gymnurus $^{c}$

C. merriami $^{b}$

C. tylorhinus ${ }^{a}$

Cryptotis goldmani

C. mexicana

C. parva

Dasypus novemcinctus

Dermanura azteca

D. phaeotis

D. tolteca

Desmodus rotundus

Didelphis marsupialis

D. virginiana

Dipodomys ordii

D. phillipsii

Eptesicus fuscus

Eumops underwoodi

Glaucomys volans

Glossophaga leachii

Habromys simulatus

Herpailurus yagouaroundi

Hylonycteris underwoodi

Lasiurus blossevillii

L. cinereus

L. intermedius

L. xanthinus

Leptonycteris curasoae

L. nivalis

Lepus californicus

Liomys irroratus

L. pictus

L. spectabilis ${ }^{c}$

Lontra longicaudis

Lynx rufus

Macrotus waterhousii

Megadontomys thomasi

Megasorex gigas

Mephitis macroura

Microtus mexicanus
Musonycteris harrisoni

Mustela frenata

Myotis auriculus

M. californicus

M. carteri

M. keaysi

M. lucifugus

M. nigricans

M. thysanodes

$M$. velifer

$M$. volans

M. yumanensis

Nasua narica

Natalus stramineus

Nelsonia goldmani ${ }^{c}$

Neotoma albigula

N. mexicana

N. micropus

N. nelsoni

Neotomodon alstoni ${ }^{a}$

Notiosorex crawfordi

Nyctinomops femorosaccus

N. macrotis

Nyctomys sumichrasti

Odocoileus virginianus

Oligoryzomys fulvescens

Oryzomys couesi

Osgoodomys banderanus

Pappogeomys bulleri

Parastrellus hesperus

Perognathus flavescens

P. flavus

Peromyscus aztecus

P. beatae

P. boylii

P. bullatus ${ }^{b}$

P. difficilis

$P$. furvus

P. gratus

$P$. levipes

P. maniculatus

P. melanotis

P. mexicanus

P. pectoralis

P. perfulvus

P. spicilegus

P. truei

Peropteryx macrotis

Philander opossum

Potos flavus

Procyon lotor

Promops centralis

Pteronotus personatus

Puma concolor

Reithrodontomys chrysopsis ${ }^{a}$ 
Apéndice 1. Continúa.

R. fulvescens

R. hirsutus ${ }^{c}$

R. megalotis

R. mexicanus

R. microdon

R. sumichrasti

Rhogeessa gracilis

R. tumida

Romerolagus diazi ${ }^{b}$

Saccopteryx bilineata

Sciurus aureogaster

S. colliaei

S. deppei

S. nayaritensis

S. oculatus

Sigmodon alleni

S. fulviventer

S. hispidus

S. leucotis

S. mascotensis

Sorex macrodon ${ }^{b}$
S. monticolus

S. oreopolus

S. saussurei

S. ventralis ${ }^{b}$

Spermophilus adocetus

$S$. mexicanus

S. perotensis ${ }^{b}$

S. variegatus

Spilogale putorius

Sturnira lilium

S. ludovici

Sylvilagus audubonii

S. cunicularius

S. floridanus

Tadarida brasiliensis

Taxidea taxus

Thomomys umbrinus

Tlacuatzin canescens

Urocyon cinereoargenteus

Xenomys nelsoni

Zygogeomys trichopus ${ }^{c}$ 